\title{
Determinação do teor de oxalato em alimentos e a sua influência desse íon no
}

\section{organismo humano}

\author{
Determination of the oxalate content in food and the influence of this ion on the human organism \\ Determinación del contenido de oxalato en los alimentos y la influencia de este ión en el organismo \\ humano
}

Recebido: 01/11/2021 | Revisado: 13/11/2021 | Aceito: 17/11/2021 | Publicado: 18/11/2021

Leandro da Silva Pinheiro

ORCID: https://orcid.org/0000-0003-4629-1280 Instituto Educacional Santa Catarina - Faculdade Guaraí, Brasil E-mail: leandropinhe1122@gmail.com

Jhennyfer Katerine Pinheiro Massoli

ORCID: https://orcid.org/0000-0001-5331-3922 Instituto Educacional Santa Catarina - Faculdade Guaraí, Brasil E-mail:Jhennyferkaterine23@gmail.com

Liberta Lamarta Favoritto Garcia Neres

ORCID: http://orcid.org/0000-0003-1327-605X Instituto Educacional Santa Catarina - Faculdade Guaraí, Brasil E-mail: liberta.neres@iescfag.edu.br

Heliane Sousa da Silva

ORCID: https://orcid.org/0000-0001-6387-5355 Instituto Educacional Santa Catarina - Faculdade Guaraí, Brasil E-mail: heliane.silva@ iescfag.edu.br

\begin{abstract}
Resumo
Sabe-se que os alimentos são essenciais para o bom funcionamento do organismo pois apresentam os nutrientes necessários para a homeostase. Entretanto, os alimentos também possuem antinutrientes, como por exemplo o oxalato de cálcio, que pode ocasionar prejuízos ao sistema urinário, como a formação de cálculos renais. Neste enfoque, o presente trabalho teve por objetivos efetuar a revisão de literatura sobre o sistema renal, a influência do oxalato no organismo humano e também determinar a concentração de oxalato presente nos extratos de tomate, batata doce e jiló. Para o levantamento bibliográfico, foram pesquisados artigos utilizando bancos de dados como Google Acadêmico, Scielo, Bireme e ScienceDirect, com os seguintes descritores em português: oxalato de cálcio, doenças renais, determinação de oxalato em alimentos e posteriormente, os mesmos descritores foram pesquisados em inglês. Para a determinação de oxalato, formam preparados extratos de tomate, jiló e batata doce, e em seguida, os extratos foram analisados pelo método de permanganometria. Os resultados mostraram que há uma relação entre formação de cálculos renais com o consumo de alimentos que apresentam oxalato, sendo possível concluir que a ingesta inadequada de água associada à uma dieta rica em alimentos que apresentam oxalato, pode contribuir com a formação de cálculos renais. Desta forma, torna-se muito importante o acompanhamento desses pacientes pelo nutricionista, a fim de efetuar uma orientação quanto à restrição do consumo desses alimentos e aconselhamento sobre a importância de se ingerir um volume adequado de água.
\end{abstract}

Palavras-chave: Oxalato de cálcio; Doenças renais; Determinação de oxalato; Fisiologia renais.

\begin{abstract}
It is known that foods are essential for the proper functioning of the body as they present the nutrients necessary for homeostasis. However, foods also have antinutrients, such as calcium oxalate, which can damage the urinary system, such as the formation of kidney stones. In this approach, this study aimed to review the literature on the renal system, the influence of oxalate on the human body and also determine the concentration of oxalate present in tomato, sweet potato and eggplant extracts. For bibliographical research, articles were searched using databases such as Google Scholar, Scielo, Bireme and ScienceDirect, with the following descriptors in Portuguese: calcium oxalate, kidney diseases, oxalate determination in food and later, the same descriptors were searched in English. For the determination of oxalate, tomato, eggplant and sweet potato extracts were prepared, and then the extracts were analyzed by the permanganimetry method. The results showed that there is a relationship between the formation of kidney stones with the consumption of foods that contain oxalate, and it is possible to conclude that the inadequate intake of water associated with a diet rich in foods that contain oxalate, can contribute to the formation of kidney stones. Thus, it is very important to monitor these patients by a nutritionist, in order to provide guidance on the restriction of consumption of these foods and advice on the importance of ingesting an adequate volume of water.
\end{abstract}

Keywords: Calcium oxalate; Kidney diseases; Determination of oxalate; Kidney physiology. 


\section{Resumen}

Se sabe que los alimentos son fundamentales para el buen funcionamiento del organismo ya que presentan los nutrientes necesarios para la homeostasis. Sin embargo, los alimentos también contienen antinutrientes, como el oxalato de calcio, que pueden dañar el sistema urinario, como la formación de cálculos renales. En este enfoque, este estudio tuvo como objetivo revisar la literatura sobre el sistema renal, la influencia del oxalato en el cuerpo humano y también determinar la concentración de oxalato presente en extractos de tomate, boniato y berenjena. Para la investigación bibliográfica se buscaron artículos en bases de datos como Google Scholar, Scielo, Bireme y ScienceDirect, con los siguientes descriptores en portugués: oxalato de calcio, enfermedades renales, determinación de oxalato en alimentos y posteriormente se buscaron los mismos descriptores en inglés. Para la determinación de oxalato se prepararon extractos de tomate, berenjena y camote, y luego se analizaron los extractos por el método de permanganimetría. Los resultados mostraron que existe una relación entre la formación de cálculos renales con el consumo de alimentos que contienen oxalato, y es posible concluir que la ingesta inadecuada de agua asociada a una dieta rica en alimentos que contienen oxalato, puede contribuir a la formación de cálculos renales. Por ello, es muy importante el seguimiento de estos pacientes por parte de un nutricionista, con el fin de orientar sobre la restricción del consumo de estos alimentos y asesorar sobre la importancia de ingerir un volumen adecuado de agua.

Palabras clave: Oxalato de cálcio; Enfermedades renales; Determinación de oxalato; Fisiología renal.

\section{Introdução}

Os alimentos são essenciais para o organismo humano pois apresentam nutrientes primordiais para o desenvolvimento e manutenção das atividades bioquímicas. Entretanto, existem uma série de fatores que interferem na absorção de vitaminas e minerais, sendo uma delas a presença de antinutrientes que limitam a biodisponibilidade dos minerais, como é o caso do oxalato (Benevides, 2013). Além dele, existem vários tipos de antinutrientes identificados como: saponinas, ácido fítico, taninos, inibidores de tripsina e nitrato (Maradini Filho, 2014).

Esses elementos antinutricionais (EANs) são substâncias presentes no metabolismo secundário das plantas, que estão presentes em diversos alimentos de origem vegetal. Os EANs ao serem ingeridos, afetam negativamente os valores nutritivos essenciais para o organismo e, quando consumidos à longo prazo, podem causar lesões graves. Se forem ingeridos em pequenas quantidades, podem apresentar efeitos benéficos para o organismo, pois atuam como antioxidantes, exercem efeito protetor do sistema circulatório e são redutores da pressão sanguínea. Por isso, esses compostos são considerados fatores nutritivamente ativos ou bioativos muito utilizados na produção de novos fármacos (Higashijima, 2020).

A biodisponibilidade dos minerais no organismo é afetada pelos fatores antinutricionais, pois essas substâncias têm a capacidade de complexar ou quelar os minerais do organismo, tornando-os insolúveis e provocando a diminuição dos nutrientes disponíveis e essenciais para corpo (Leal, 2010). O ácido fítico ao ligar-se com substâncias metálicas se torna insolúvel, e os taninos têm a capacidade inibir enzimas importantes que ajudam manter o funcionamento adequado do metabolismo (Oliveira, 2010).

O oxalato é um íon que se encontra nos vegetais e nos alimentos industrializados e tem complexa capacidade de ligarse ao cálcio, no qual é pouco solúvel na urina, formando o cálculo renal. O oxalato é encontrado em três maneiras distintas no organismo, que são as formas monoidratada, di-hidratada e tri-hidratada (Souza Neto, 2017).

Sabe-se que os rins são necessários para a manutenção do organismo e é o principal órgão regulador da homeostase do corpo. Quando uma substância afeta o processo renal, as suas funções acabam sendo prejudicadas. Neste enfoque, o oxalato proveniente dos alimentos, pode reagir com o cálcio formando-se cristais não solúveis, promovendo uma obstrução do trato urinário sendo possível evoluir para uma nefrolitíase. A presença dessa doença pode ser diagnosticada através do exame de urina para analisar a existência de oxalato de cálcio (Gordiano, 2014).

O sistema renal reconhece quando há excesso de água ou eletrólitos específicos e reagem cessando a reabsorção ou secretando substâncias, regulando assim o balanço hídrico, eletrolítico e ácido-base. Nos rins são produzidos e liberados hormônios que equilibram a pressão arterial, a produção de eritropoietina e a síntese da vitamina D (Souza, et al., 2021). 
A obesidade e o sobrepeso são considerados fatores de riscos no desenvolvimento para doença de carcinoma, particularmente associado com doenças renais, cálculos de ácido úrico e oxalato de cálcio (Silva Junior, et al., 2017).

O ácido úrico é considerado um ácido orgânico fraco, cujo valor numérico de pKa igual a 5,5 na urina. Se o pH urinário de um indivíduo diminuir, pode ocorrer o aumento da formação de cálculos renais de ácido úrico, pois facilita o processo de cristalização desta substância (Carvalho, 2018).

Já a formação dos cálculos de oxalato, está diretamente relacionada com as quantidades urinários de cálcio, oxalato, urato e também inversamente relacionada com as concentrações de magnésio e citrato. Cerca de $80 \%$ dos casos de cálculos renais são compostos por oxalato de cálcio, 1 a $10 \%$ são cálculos de fosfato de cálcio, $10 \%$ são cálculos de estruvita (fosfato triplo amoníaco magnesiano), $9 \%$ são cálculos de ácido úrico e $1 \%$ são cálculos de cistina, uratos ou relacionados à fármacos (Sartarelli, 2018).

O cálcio faz parte de um grupo de minerais essenciais que apresenta maiores concentrações no organismo, sendo responsável por $1 \%$ a $2 \%$ do peso corporal e $99 \%$ se encontra nos ossos e dentes, sob a forma de cristais de hidroxiapatita, o que garante a sustentação do esqueleto e a manutenção da concentração de cálcio sérico. Ele pode ser adquirido por meio da ingesta de alimentos como o leite, queijo, manteiga, iogurte e seus derivados (Premaor, et al., 2016 \& Campos et al., 2013).

Quando uma pessoa efetua um consumo inadequado de água, o cálcio juntamente com o oxalato, provenientes da alimentação, podem se precipitar na forma de oxalato de cálcio formando os cálculos renais, tornando-se primordial saber a concentração de oxalato presente nos alimentos. Neste sentido, esse trabalho teve por objetivos determinar o teor de íon oxalato nos extratos de tomate, de jiló, da batata-doce e verificar a influência do oxalato de cálcio na formação de cálculo renais.

\section{Materiais e Métodos}

O presente trabalho foi dividido em duas etapas, sendo a primeira parte de revisão de literatura sobre o oxalato e sua relação com o organismo humano e a segunda parte experimental, onde foi determinada a concentração de oxalato nos extratos de tomate, jiló e batata-doce.

Para o referencial teórico foram pesquisados artigos, dissertações de mestrado e teses de doutorado entre os anos de 2005 a 2021, utilizando-se os bancos de dados do Google Acadêmico, Scielo, Bireme e ScienceDirect, com os seguintes descritores em português: oxalato de cálcio, doenças renais, determinação de oxalato em alimentos e posteriormente os mesmos descritores foram pesquisados em inglês.

Os artigos científicos e demais referencias teóricos foram organizados em tabelas e após a leitura minuciosa, foram selecionados para a execução deste trabalho, sendo excluídos aqueles que fugiam do tema.

\section{Procedimento experimental}

\section{Aquisição e preparo das amostras}

Os alimentos utilizados no desenvolvimento deste trabalho foram adquiridos no município de Guaraí-TO, acondicionados em sacolas plásticas limpas e em seguida foram levados para o Laboratório de Química para a realização das análises.

As amostras foram lavadas com água destilada e foram secas com papel toalha. Posteriormente, foram pesadas, cordadas em pedaços e maceradas em cadinho de porcelana. Transferiu-se o material para um béquer de 1,0 L, adicionou-se $50 \mathrm{~mL}$ de solução de $\mathrm{H}_{2} \mathrm{SO}_{4}$ 2,0 M e o sistema conduzido ao Banho-Maria, à $60{ }^{\circ} \mathrm{C}$, durante uma hora para a extração do ácido oxálico $\left(\mathrm{H}_{2} \mathrm{C}_{2} \mathrm{O}_{4}\right)$. $\mathrm{O}$ material obtido foi filtrado em um Erlenmeyer e foi acondicionado na geladeira, à $8{ }^{\circ} \mathrm{C}$, até o momento do uso, conforme é mostrado na Figura 1. 
Figura 1: preparo da amostra de tomate.

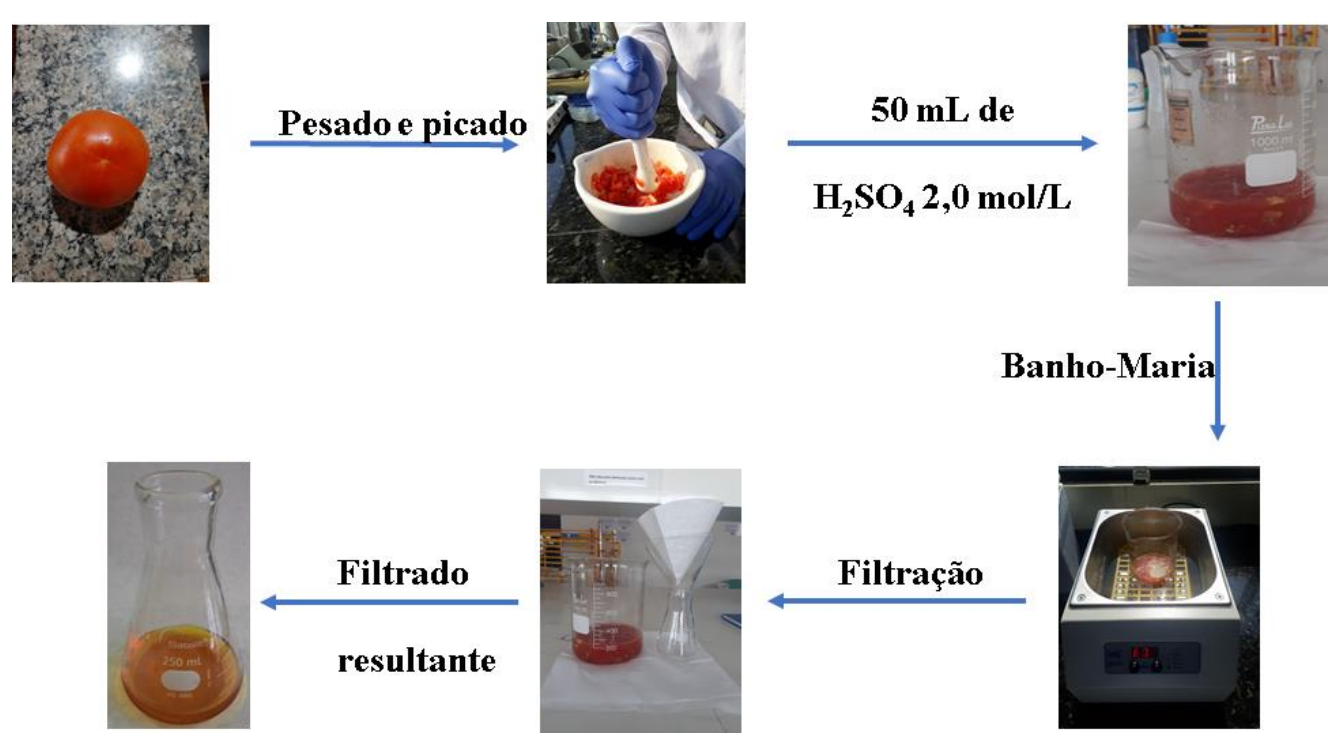

Fonte: Autores (2021).

Foram pipetados 1,0 mL do filtrado resultante e transferido para um balão volumétrico de $50 \mathrm{~mL}$. Em seguida, completouse com solução de $\mathrm{H}_{2} \mathrm{SO}_{4} 2,0 \mathrm{~mol} / \mathrm{L}$ até o menisco. Os testes foram realizados em quintuplicadas, as soluções preparadas foram transferidas para os Erlenmeyers e identificados.

Para o preparo das outras amostras de jiló e da batata-doce se procedeu da mesma forma para as amostras de tomate.

\section{Quantificação do teor de oxalato}

Para as determinações do teor de oxalato foi utilizado o método de permanganometria, onde foi preparada uma solução padronizada de permanganato de potássio $\left(\mathrm{KMnO}_{4}\right)$ 0,02 mol/L para titular as soluções contendo as amostras. $\mathrm{O}$ ponto final da titulação foi quando a solução incolor mudou para uma tonalidade róseo claro e perdurou por mais de 30 segundos.

\section{Revisão de Literatura}

\section{Anatomia e fisiologia do sistema renal}

Sabe-se que os rins são necessários para a manutenção do organismo e é o principal órgão regulador da homeostase do corpo. Segundo Albuquerque (2017), o sistema urinário é formado por dois rins, dois ureteres, uma bexiga e uma uretra (Figura 2 A). Portanto, os rins são órgãos pares de coloração marrom-avermelhado, com a forma de um grão de feijão (figura 2 B), cobertos por uma membrana fibromuscular fina e brilhante chamada de cápsula renal. Estão localizados paralelamente à coluna vertebral, sendo que o rim direito é ligeiramente mais baixo do que o esquerdo, porque o fígado ocupa um espaço considerável, no lado direito, acima do rim. 
Figura 2: (A) Sistema renal. (B) Os principais componentes dos rins.

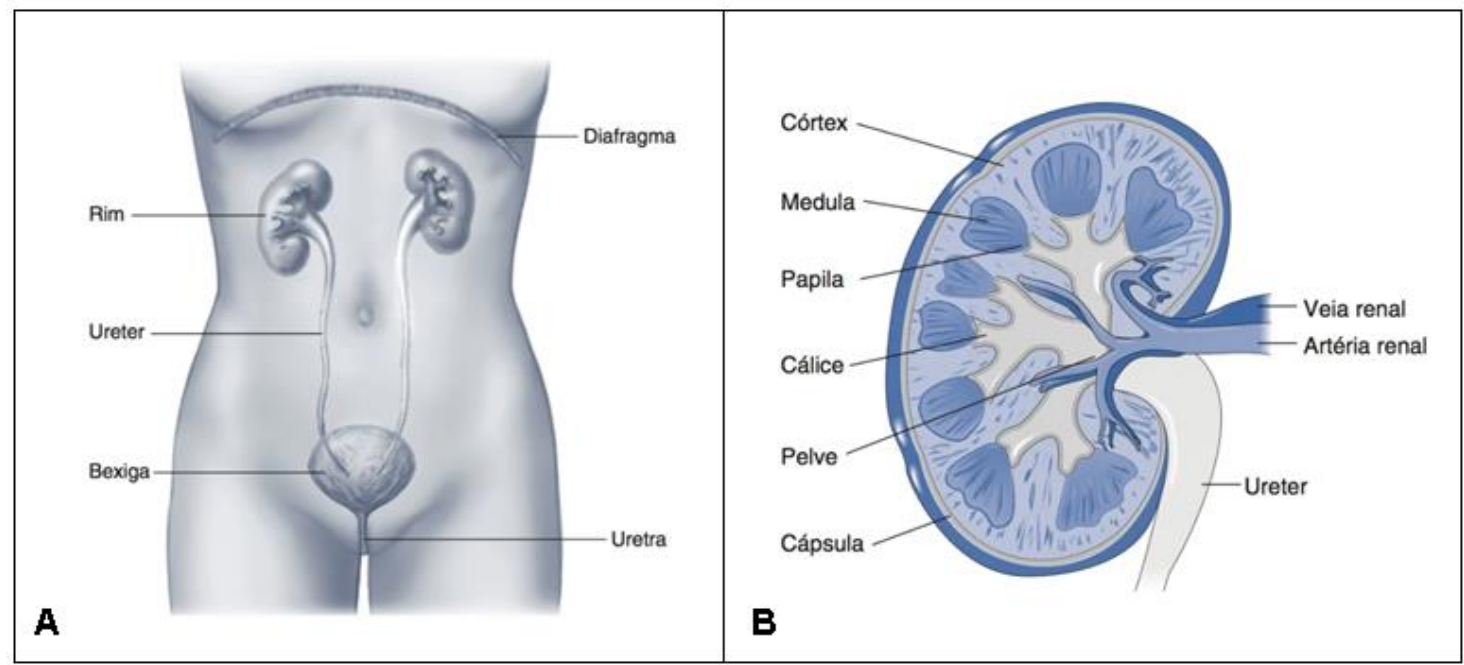

Fonte: Eaton \& Pooler (2016).

A principal parte funcional do rim é o néfron, onde em cada rim apresenta cerca de 1000000 néfrons. O sangue da arteríola aferente entra no glomérulo, onde parte é filtrado e parte sai do glomérulo pela arteríola eferente. O ultrafiltrado glomerular passa pelos túbulos do néfron, onde ocorre seu processamento através de reabsorção. O sistema renal tem como principais funções: excreção de metabólitos como: ureia, creatinina, ácido úrico, toxinas e fármacos, controle hídrico através da diurese e controle intra e extracelular, no processo de regulação através da produção de hormônios e no controle ácido básico do sangue (Teixeira, 2021).

No sistema renal é produzido a prostaglandina, que é sintetizada nos túbulos por indução da COX-2, tem papel essencial na reabsorção de água através da modulação dos efeitos da vasopressina, no transporte de cloreto de sódio na alça de Henle e no aumento da secreção de potássio. A prostaglandinas com ação da COX-1 são encarregadas pela regulação da hemodinâmica renal, da taxa de filtração glomerular (TFG) e se encontra associada com a manutenção do equilíbrio hidroeletrolítico, através do controle da excreção de água e íons (Silva, 2014).

Os túbulos proximal e distal, alça de Henle e o ducto coletor são responsáveis de reabsorver e secretar íons para ocorrer um equilíbrio homeostático que é regulado por hormônios, destacando-se o sistema renina-angiotensina-aldosterona e o hormônio antidiurético (ADH), além de outras substâncias, como o óxido nítrico (Santos, et al., 2017).

Os derivados sintéticos de testosterona são esteroides que possuem propriedades anabólicas e podem ser empregados na terapêutica. Entretanto, o uso indiscriminado desses compostos, podem causar alterações no sistema hepático e renal, como desnaturação proteica, deposição de fibrina, hemorragia nos túbulos distais, insuficiência renal, necrose tubular aguda caracterizada pelo inchaço de células tubulares e a nefrocalcinose, que ocorre com um aumento da deposição de cálcio nos rins, ocasionando nefrolitíase (Costa, et al., 2020).

\section{A influência do íon oxalato no metabolismo do cálcio}

O íon oxalato é o principal componente para formação dos cálculos, razão pela qual especialistas indicam a restrição desse íon na dieta de pessoas com doenças renais. Quando se compara as alterações das concentrações de cálcio e de oxalato na formação de cálculo, se observa que pequenas variações na concentração de oxalato, ocorre maiores alterações sobre a cristalização de oxalato de cálcio do que para grandes alterações na concentração de cálcio no organismo. Porém, esta interação de cálcio e oxalato necessita ficar constantemente equilibrado, para formação do cálculo na urina (Mello, 2006). 
Durante a vida, a absorção do cálcio no organismo varia conforme a faixa etária e as condições do indivíduo. No aspecto geral, quanto maior a necessidade e menor o fornecimento dietético de cálcio, mais eficiente será a absorção em cada pessoa. De acordo com o crescimento, gravidez no caso de mulheres, lactação, deficiência de cálcio e na atividade física que resulta em alta densidade óssea, ocorre uma maior intensificação da absorção desse mineral pelo organismo (Buzinaro, et al., 2006).

Vale a pena mencionar que a Síndrome Metabólica (SM) e a obesidade são associadas com pH urinário ácido e podem apresentar fatores de risco para cálculos renais. Cerca de $80 \%$ dos cálculos são compostos de cálcio que se ligam ao oxalato formando um precipitado insolúvel (Tessaro, et al., 2018). O pH urinário associado com casos de cálculos de oxalato de cálcio pode ser controverso, de acordo com a maioria dos autores, os cálculos desse mineral podem se formar independentemente do valor de pH urinário (Carvalho, 2018).

Segundo Sartarelli (2018), a supersaturação urinária em oxalato, influenciada significativamente pelo oxalato dietético e também pela produção endógena, é um fator importante na formação dos cálculos. Não há uma terapia estabelecida que diminua a concentração urinária de oxalato, neste caso, os pacientes são orientados a evitar o consumo de alimentos ricos em oxalato.

Quando uma substância afeta o processo renal, as suas funções acabam sendo prejudicadas. Neste enfoque, o oxalato proveniente dos alimentos reage com o cálcio formando-se cristais não solúveis, promovendo uma obstrução do trato urinário no qual pode evoluir para uma nefrolitíase. A presença dessa doença pode ser diagnosticada através do exame de urina para analisar a existência de oxalato de cálcio (Gordiano, 2014).

\section{A influência do oxalato e a formação do cálculo renal nas pessoas}

A formação dos cálculos está diretamente relacionada com as quantidades urinários de cálcio, oxalato e urato e, também inversamente relacionada, com as concentrações de magnésio e citrato. Cerca de $80 \%$ dos casos de cálculos renais são compostos por oxalato de cálcio, 1 a $10 \%$ são cálculos de fosfato de cálcio, $10 \%$ são cálculos de estruvita (fosfato triplo amoníaco magnesiano), 9 \% são cálculos de ácido úrico e $1 \%$ são cálculos de cistina, uratos ou relacionados à fármacos (Sartarelli, 2018).

Os cálculos de estruvita são formados de magnésio, amônio e fosfato produzidas por meio de algumas infecções no aparelho urinário. Estas infecções são causadas por bactérias como: Pseudomonas, Staphylococcus, Proteus e outras. Devido a ação da uréase bacteriana ocorre a produção de amônia a partir da ureia, fazendo com que a urina se torne extremamente alcalina e propícia para a formação dos cristais do tipo estruvita (Salem, 2019).

Há vários fatores associados com a formação de cálculos renais como hereditariedade, obesidade, fatores climáticos e sazonais, sedentarismo, baixa ingestão de líquidos, fatores epidemiológicos, imobilização prolongada, utilização de drogas litogênicas, diminuição do volume de urina, distúrbios metabólicos, alterações do pH da urina e dieta que favoreça a formação do cálculo (Lopata, et al., 2017).

O consumo de proteínas animais e de sódio são fatores de riscos que podem aumentar a formação de cálculos renais. O sódio pode provocar hipercalciúria devido à troca tubular renal com o cálcio, já as proteínas provocam uma sobrecarga ácida, influenciando a composição da urina ao aumentar a excreção de cálcio e oxalato, diminuindo a excreção de citrato (Fernandes, 2018). A diminuição do volume diário de urina é um outro fator que contribui para a formação de cálculos, ainda mais quando os valores são menores do que um litro por dia, o risco de desenvolvimento dos cálculos aumenta (Furlanetto, 2018).

Segundo Lopata e colaboradores (2017), inicialmente acontece a saturação da urina no sistema renal, posteriormente, ocorre o processo de nucleação com formação dos cristais, que são liberados livremente na urina. Todavia, na presença de alguns fatores que ajuda no processo, ocorre a agregação de cristais, com a junção dos cristais recém-formados, formando aglomerados pelo meio de forças intermoleculares. Na etapa final, ocorre capturas e as partículas aglomeradas ficam pressas no sistema 
coletor. A formação dos cálculos ocorrerá se as partículas capturadas aumentarem de tamanho devido à processos físico-químicos envolvidos na saturação urinária, levando ao aumento da litíase urinária.

\section{Resultados e Discussão}

O permanganato é um excelente agente oxidante e, em soluções com pH baixo, apresenta coloração violeta e é reduzido à $\mathrm{Mn}^{2+}$, fazendo com que o sistema mude de cor ficando incolor. A reação que mostra esse processo químico (Skoog, 2006) se encontra na equação 1 .

$$
2 \mathrm{MnO}_{4}^{-}(\mathrm{aq})+5 \mathrm{H}_{2} \mathrm{C}_{2} \mathrm{O}_{4(\mathrm{aq})}+6 \mathrm{H}_{(\mathrm{aq})}^{+} \rightarrow 2 \mathrm{Mn}^{2+}{ }_{(\mathrm{aq})}+10 \mathrm{CO}_{2(\mathrm{~g})}+8 \mathrm{H}_{2} \mathrm{O}_{(\mathrm{l})} \quad \text { Eq. } 1
$$

Quando se efetua a titulação da amostra com permanganato em meio ácido, tem-se uma descoloração da solução. O ponto final da titulação ocorreu quando passou de incolor para levemente rosa, onde todo oxalato foi oxidado. Os volumes gastos de permanganato utilizados para titular as amostras foram anotados, em seguida foram calculadas as concentrações de oxalato e os resultados foram expressos em mg de oxalato/100 g de amostra, conforme a Figura 3.

Figura 3: Teor de oxalato nos extratos de tomate, de batata-doce e de jiló expressos em mg de oxalato/100 g de amostra.

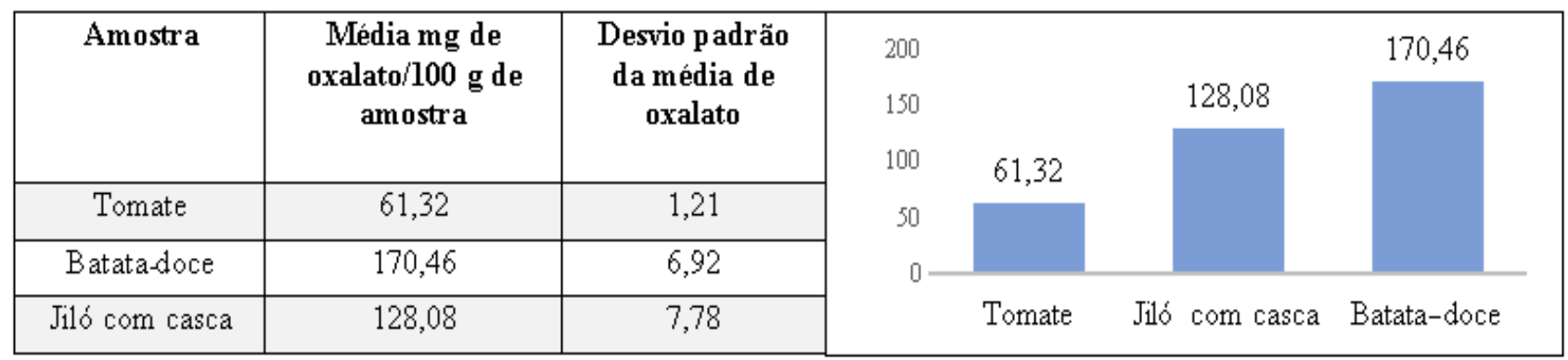

Fonte: Autores (2021).

Pode-se observar que o teor de oxalato para o extrato de batata-doce $(170,46 \mathrm{mg} \pm 6,92)$ foi maior quando comparado com o do tomate $(61,32 \mathrm{mg} \pm 1,21)$ e do jiló com casca que foi de 128,08 $\mathrm{mg} \pm 7,78$. Essa diferença entre os teores de oxalato encontrados pode ser explicada devido aos diferentes tipos de solos onde os alimentos foram cultivados, tipos de clima e diferenças da maturação no caso dos frutos (tomate e jiló) (Liebman, 2012).

Segundo Oliveira e colaboradores (2017), a concentração ideal de oxalato por dia a ser consumida seria de 50 a 200 $\mathrm{mg}$ e a dose letal desse íon é de $1500 \mathrm{mg}$. Desta forma, deve-se ter cuidado ao consumir alimentos com elevados teores de oxalato como é o caso da batata-doce, conforme mostraram os resultados.

O oxalato é um ânion bivalente que pode provocar irritação do trato gastrintestinal. Ele interfere negativamente na biodisponilidade do íon cálcio $\left(\mathrm{Ca}^{2+}\right)$, pois pode formar cristais insolúveis de oxalato, consequentemente, pode provocar uma diminuição da concentração desse mineral no organismo, diminuindo assim a taxa de coagulação sanguínea (Lopes, et al., 2009).

A urina é uma solução supersaturada de oxalato de cálcio sob condições adequadas. Porém, quando se efetua uma dieta rica em oxalato e ao mesmo tempo efetua-se uma ingestão inadequada de água, a solubilidade do oxalato diminui, provocando a precipitação de cristais de oxalato, formando os chamados cálculos renais (Oliveira, 2017). Assim, para ocorrer uma diminuição de urolitíase em pacientes com disfunção renal, o indivíduo adulto deve ingerir em torno de 1,5 L a 3,0 L de água por dia, dependendo da sua massa corporal (Carvalho, 2010), para promover um efeito de diluição da urina (Furlanetto, 2018). 
Com a finalidade de diminuir a concentração de oxalatos presentes nos alimentos de origem vegetal, orienta-se colocar os alimentos na presença de água, fervê-los e não ingerir essa água utilizada no processo, devendo-se efetuar o descarte da mesma. Um outro fator interessante para diminuir a absorção do oxalato pelo organismo, seria consumir alimentos ricos em cálcio e em magnésio juntamente com os alimentos que apresentam oxalato, pois, esses íons formam sais insolúveis de oxalato no intestino, sendo excretados pelo corpo (Higashima, et al., 2020).

Sabe-se que alguns microrganismos que compõem a microbiota intestinal conseguem degradar o oxalato, como por exemplo o Oxalobacter formigenes, Streptococcus thermophilus e Enterococcus faecalis. Entretanto, o uso indiscriminado de antibióticos pode diminuir esses microrganismos habituais do intestino, podendo culminar na diminuição da degradação de oxalato. Em virtude disso, estudos mostraram que indivíduos que apresentam baixas concentrações de Oxalobacter formigenes, foi associado com o aumento da excreção de oxalato na urina (Hatch, 2005).

\section{Considerações Finais}

Por meio deste trabalho foi possível concluir que o teor de oxalato encontrado no tomate e no jiló foram menores em comparativo com o da batata-doce. Alimentos que apresentam elevados teores desse íon, podem afetar a biodisponibilidade do cálcio no organismo, que associada com a ingesta inadequada de água, pode facilitar a formação de cálculos renais.

Desta forma, torna-se muito importante o acompanhamento desses pacientes pelo nutricionista, a fim de efetuar uma orientação adequada quanto à restrição do consumo de alimentos que apresentam alta concentração de oxalato e aconselhamento sobre a importância de se ingerir um volume adequado de água, colaborando assim, para evitar a recorrência em pessoas com predisposição genética ao desenvolvimento de cálculos renais.

Como ainda não existe um método eficaz para a retirada do oxalato do organismo, recomenda-se a diminuição da concentração desse íon na dieta, efetuando-se o remolho dos alimentos de origem vegetal em água fervente para a extração de oxalato e posteriormente o descarte desse líquido. Tal procedimento ajuda na diminuição do oxalato presente no alimento.

Como sugestão para trabalhos futuros, propõe-se a determinação de fatores antinutricionais, que se encontram presentes em diversos alimentos de origem vegetal, como saponinas, ácido fítico, inibidores de tripsina, nitrato e verificar a influência desses compostos no organismo humano.

\section{Referências}

Albuquerque, J. M. (2017). Marcadores laboratoriais utilizados no diagnóstico da doença renal crônica. Monografia (Graduação no Curso de Bacharelado em Farmácia), Centro de Educação e Saúde, Universidade Federal de Campina Grande.

Benevides, C. D. J., Souza, R. D. B., Souza, M., Silva, M. (2013). Efeito do processamento sobre os teores de oxalato e tanino em maxixe (Cucumis anguria L.), JILÓ (Solanum gilo), feijão verde (Vigna unguiculata (L.) e feijão andu (Cajanus cajan (L.) Mill SP). Alimentos e Nutrição Araraquara. $24(3)$, p. 328.

Buzinaro, E. F., Almeida, R. N., \& Mazeto, G. M. (2006). Biodisponibilidade do cálcio dietético. Arquivos Brasileiros de Endocrinologia \& Metabologia. 50, p. 852-861. doi: https://doi.org/10.1590/S0004-27302006000500005.

Carvalho, A. P. L., \& Zanardo, V. P. S (2010). Consumo de água e outros líquidos em adultos e idosos residentes no município de Erechim - Rio Grande do Sul. Perspectiva Erechim. 34(125), p. 117-126.

Carvalho, M. (2018). pH urinário em formadores de cálculos de oxalato de cálcio: isso importa. Brazilian Journal of Nephrology. $40(1)$, 6-7.

Costa, R. L. A. \& de Melo, A. T. (2020). Disfunções anatomo-fisiológicas provenientes do uso indiscriminado de derivados sintéticos da testosterona (EAAs). Brazilian Journal of Development. 6(12), 94256-94268. doi: https://doi.org/10.34117/bjdv6n12-043.

Campos, F. S., \& Pinhati, F. R. (2013). O Controle do Cálcio e a Hipocalcemia. Cadernos UniFOA. 8(23), 77-85.

Eaton, D. C. \& Pooler, P. J. (2016). Fisiologia Renal de Vander. Porto Alegre: McGraw-Hill.

Fernandes, M. B. M. (2018). Plano de ação para diminuição da ocorrência de litíase renal na área da Equipe de Saúde da Família Luxemburgo, no município de Sete Lagoas/Minas Gerais. Monografia (Especialização em Estratégia e Saúde da Família), Universidade Federal de Minas Gerais.

Furlanetto, R. (2018). Perfil de pacientes internados por urolitíase em um hospital do norte do Estado do Rio Grande do Sul. Monografia (Graduação em Medicina), Universidade Federal da Fronteira do Sul. 
Gordiano, É. A., Tondin, L. M., Miranda, R. C. D., Baptista, D. R., \& Carvalho, M. (2014). Avaliação da ingestão alimentar e excreção de metabólitos na nefrolitíase. Brazilian Journal of Nephrology. 36, 437-445.

Hatch, M. \& Freel, R.W. (2005). Intestinal transport of an obdurate anion: oxalate. Urological Research.33(1),1-16.

Higashijima, N. S., Lucca, A., Rebizz, L. R. H., \& Rebizzi, L. M. H. (2020). Fatores antinutricionais na alimentação humana. Segurança Alimentar e Nutricional. 27, p. 1-16. doi: http://dx.doi.org/10.20396/san.v27i0.8653587.

Leal, A., \& Goncalvez, C. (2010). Avaliação da concentração de minerais e dos fatores antinutricionais fitato e oxalato em multimisturas da Região Metropolitana de Belo Horizonte/MG. Revista Sociedade Brasileira de Alimentos e Nutrição. 35(2), 39-52.

Liebman, M., Wahsh, I. A. A., \& Wu, Y. (2012). A Comparison of Two Extraction Methods for Food Oxalate Assessment. Journal of Food Research. 1(2), 233-239.

Lopata, V. J., Hofelmann, D. A., Spezia, J., \& Hauser, A. B. (2017). Análise de dados clínicos e laboratoriais associados à litíase urinária em pacientes de um laboratório de análises clínicas. Visão Acadêmica. 17(3), 18-28.

Lopes, C. O., Dessimoni, G. V., Silva, M. C., Vieira, G., \& Pinto, N. A. V.D (2009). Aproveitamento, composição nutricional e antinutricional da farinha de quinoa (Chenopodium Quinos). Alimentos e Nutrição Araraquara. 20(4), 669-675.

Maradini Filho, A. M. (2014). Caracterização físico-química, nutricional e fatores antinutricionais de quinoa da variedade brasileira BRS Piabiru. Tese (Doutoramento em Ciência e Tecnologia de Alimentos), Instituto de Ciência e Tecnologia de Alimentos, Universidade Federal de Viçosa.

Mello, E. D., \& Schneider, M. A. O. (2006). A importância da dieta no manejo da hipercalciúria. Clinical \& Biomedical Research. $26(2), 52-60$.

Oliveira, K. B. (2010). Métodos de redução e inativação dos fatores antinutricionais. Monografia (Especialização em Nutrição Humana e Saúde), Departamento de Ciências dos Alimentos, Universidade Federal de Lavras.

Oliveira, L. C. S., Kamonsec, D. H., \& Rostelato-Ferreira, S (2017). Determinação dos teores de ácido oxálico em diferentes amostras de tomate. NutrivisaRevista de Nutrição e Vigilância em Saúde. 4 (2), 61-65.

Premaor, M. O., \& Brondani, J. E. (2016). Nutrição e saúde óssea: a importância do cálcio, fósforo, magnésio e proteínas. Revista da AMRIGS. 60(3), 253-263.

Silva Junior, J. B., Bentes, A. C. S. N., Daher, E. F., \& Matos, S.M. A. (2017). Obesidade e doença renal. Jornal Brasileiro de Nefrologia. 39 (1), $65-69$.

Souza Neto, F. E. D. (2017). Caracterização e avaliação do potencial da quitosana extraída de Cunninghamella elegans na formação de cristais de oxalato de cálcio e atividade antioxidante. Dissertação (Mestrado em Ciência e Engenharia de Materiais), Universidade Federal Rural do Semiárido.

Sartarelli, N. C. (2018). Estudo do teor de ácido oxálico em hortaliças e frutos, seu potencial de cristalização in vitro e caracterização morfológica de cristais de oxalato de cálcio. Dissertação (Mestrado em Alimentos e Nutrição), Universidade Estadual Paulista.

Salem, P. P. D. O. (2019). Fitoquímica, metabolômica e avaliação anti-inflamatória e antiurolítica de Cissus gongylodes. Dissertação (Mestrado em Química), Universidade Federal de Alfenas.

Santos, H. A. L. D., Silva, C. A. F., \& Aragão Neto, A. (2017). Tratamento odontológico em paciente com insuficiência renal crônica. Monografia (Graduação em Odontologia), Faculdade Integrada de Pernambuco.

Silva, J. M., Mendonça, P. P., \& Partata, A. K. (2014). Anti-inflamatórios não-esteróides e suas propriedades gerais. Revista Científica do ITPAC. 7(4), 1-15.

Skoog, D. A.; West, D. M., Holler, F. J, Crouch, S. R (2006). Fundamentos de Química Analítica. São Paulo: Senarg Learning, 1026 p.

Tessaro, C. Z. W., Ramos, C. I., \& Heilberg, I. P. (2018). Influência do estado nutricional e dos parâmetros laboratoriais e dietéticos sobre a excreção urinária ácida em pacientes portadores de litíase cálcica. Brazilian Journal of Nephrology, 40, 35-43.

Teixeira, D. A. (2021). Fisiologia humana. Teófilo Otoni: UNIPAC, 73 p. 